\title{
Gauge symmetry and non-abelian topological sectors in a geometrically constrained model on the honeycomb lattice
}

\author{
Paul Fendley ${ }^{1}$, Joel E. Moore ${ }^{2,3}$, and Cenke $\mathrm{Xu}^{2}$ \\ 1 Department of Physics, University of Virginia, \\ Charlottesville, VA 22904-4714 USA \\ 2 Department of Physics, University of California, \\ Berkeley, CA 94720 \\ 3 Materials Sciences Division, Lawrence Berkeley National Laboratory, \\ Berkeley, CA 94720 \\ (Dated: January 17, 2007)
}

\begin{abstract}
We study a constrained statistical-mechanical model in two dimensions that has three useful descriptions. They are 1) the Ising model on the honeycomb lattice, constrained to have three up spins and three down spins on every hexagon, 2) the three-color/fully-packed-loop model on the links of the honeycomb lattice, with loops around a single hexagon forbidden, and 3) three Ising models on interleaved triangular lattices, with domain walls of the different Ising models not allowed to cross. Unlike the three-color model, the configuration space on the sphere or plane is connected under local moves. On higher-genus surfaces there are infinitely many dynamical sectors, labeled by a noncontractible set of nonintersecting loops. We demonstrate that at infinite temperature the transfer matrix admits an unusual structure related to a gauge symmetry for the same model on an anisotropic lattice. This enables us to diagonalize the original transfer matrix for up to 36 sites, finding an entropy per plaquette $S / k_{B} \approx 0.3661 \ldots$ and substantial evidence that the model is not critical. We also find the striking property that the eigenvalues of the transfer matrix on an anisotropic lattice are given in terms of Fibonacci numbers. We comment on the possibility of a topological phase, with infinite topological degeneracy, in an associated two-dimensional quantum model.
\end{abstract}

\section{INTRODUCTION}

Classical lattice statistical-mechanical models with local constraints have been of great interest for decades. By "local constraint", we mean a local rule which restricts the allowed configurations. A famous example is that of the hardcore close-packed dimer model ${ }^{1,2}$. The degrees of freedom are dimers stretching between adjacent sites of a lattice, while the hard-core and close-packing constraints mean that each site of the lattice is touched by exactly one dimer. Another famous example is Baxter's three-color model, where each link is covered by one of three "colors" of dimers, with the constraint that each site is touched by all three colors ${ }^{3}$.

Another oft-studied constraint is to require that the degrees of freedom be "loops", i.e. one-dimensional objects without ends. For example, both close-packed hard-core dimers and the three-color model can be viewed as loop models. In the latter case, the links colored by two of the colors (say $R$ and $G$ ) form closed loops of alternating $R$ and $G$ colors. Since every vertex has one $R$ and one $G$ touching, the three-color model is therefore equivalent to a fully-packed loop model (every site has one loop going through it). Each loop receives a weight 2 , since there are two possible ways of ordering $R$ and $G$ around each loop.

One interesting limit of constrained models is at infinite temperature, where each allowed configuration has the same Boltzmann weight. The partition function in this limit is a purely combinatorial quantity: it simply counts the number of configurations. Because of the constraints, the physics of such models is still very rich. For example, the three-color model is critical, as is the hard-core close-packed dimer model on the square lattice ${ }^{2}$. Obviously, not all constrained models are critical: dimers on the triangular lattice (or any non-bipartite lattice) have exponentially decaying correlators ${ }^{4}$.

The purpose of this paper is to present a constrained lattice model that has several rather interesting properties. There are three equivalent ways of defining the model. One is as an Ising model on the honeycomb lattice with a constraint around each hexagon; one is with a constraint on the three-color model, and a third is as three coupled Ising models. The latter form is most naturally given in terms of loops representing Ising domain walls, and is also the representation where its properties are most transparent.

This model is of interest for several reasons. It is defined in terms of simple local degrees of freedom and constraints, yet exhibits fascinating conservation laws. As we will detail, the transfer matrix decomposes into sectors which are labeled by non-abelian (and non-local) charges. The number of distinct sectors exponentially increases as the size of the system increases. This symmetry enables us to do exact diagonalization of the transfer matrix for systems of sizes up to 36 sites across, i.e. a Hilbert space initially of size $2^{36}$. We know of no non-trivial system with such a property.

Despite the fact that the conserved charges are non-local, the configuration space of the model has the striking property that it is connected under simple local moves. Even though the three-color model is closely related to ours, to relate all its different configurations requires changing degrees of freedom arbitrarily far apart ${ }^{5,6}$. Since we give a precise relation between the three-color model and ours, we thus have located the obstruction to connectivity (the "11th" vertex discussed below) in the three-color model. Not only does this mean that 
our model is amenable to Monte Carlo situations, but it should prove interesting to study its classical dynamics 5,7 .

A new reason to be interested in two-dimensional classical lattice models with constraints comes from quantum physics. The motivation is to find phases with topological order, where there is no non-vanishing local order parameter, but only nonlocal ones. The idea for building such a quantum model by starting with a classical magnet with local constraints came long $\mathrm{ago}^{8}$, and a theoretical triumph in proving they exist came from a quantum eight-vertex $\operatorname{model}^{9}$ and a quantum dimer model on the triangular lattice 4 . The two-dimensional quantum models are defined by using each configuration in a two-dimensional classical lattice model as a basis element of the Hilbert space. One characteristic of topological order is that the number of ground states depends on the genus of twodimensional space. Constrained lattice models give natural ways of defining the different sectors which, with appropriate choice of Hamiltonian ${ }^{10}$, correspond to different ground states in the quantum theory. When writing the eight-vertex or dimer models as loop models, the different ground states are labeled by the number $(\bmod 2)$ of loops which wrap around cycles of the torus.

In section $\Pi$ we introduce these models and show that they are equivalent. We relate our model to several others in appendix A enabling us to put upper and lower bounds on the entropy. In section III, we discuss the dynamics under local moves, showing that the configurations on the plane or sphere are all connected by simple local moves. On surfaces with non-contractible cycles, we classify the infinitely many separate dynamical sectors. In section IV, we give arguments which suggest our model is not critical. We also develop in section $\mathrm{V}$ and solve in section $\mathrm{VI}$ a closely related model which has a gauge symmetry. We exploit this symmetry in section VII to show how to reduce dramatically the size of the original transfer matrix. This enables us to exactly diagonalize the transfer matrix for quite large lattices, and the results again suggest that the model is not critical. In section 9, we present our conclusions, and discuss applying our results to build a quantum model with topological order.

\section{THE MODEL, AND ITS THREE DESCRIPTIONS}

The model we are introducing can be described in three equivalent ways. Here we present them, and then demonstrate their equivalence.

a. Model 1: The degrees of freedom of the Ising model are "spins" $\sigma_{i}$ taking values of \pm 1 at each site $i$ of some lattice. The energy in general from nearest-neighbor interactions is given by

$$
E=J \sum_{\langle i j\rangle} \sigma_{i} \sigma_{j}
$$

The Ising model on the honeycomb lattice has a critical point when $K=J / k_{B} T=\operatorname{arcsinh}(\sqrt{3}) / 2^{11}$. Our model 1 is the Ising model on the honeycomb lattice, with the constraint that there must be three up spins and three down spins around each hexagon, i.e.

$$
\text { model 1: } \quad M_{h} \equiv \sum_{i \in \square} \sigma_{i}=0
$$

This is quite a strong constraint, retaining only 20 of the original 64 possibilities for the spins around each hexagon. We will mostly discuss the infinite temperature limit $T \rightarrow \infty$ or $K=0$, in which each allowed configuration has equal weight.

b. Model 2: The degrees of freedom in the three-color model are three colors, say $R, G$, and $B$, which are placed on the links on the honeycomb lattice. The usual constraint in the three-color model is to require that at each site of the lattice, all three colors appear. In other words, links of the same color can never touch. When the partition function is simply the sum over all allowed configurations (i.e. in the infinitetemperature limit), the model is critical and integrable ${ }^{3}$. Our model 2 is the three-color model with an additional constraint forbidding configurations which have the same two colors alternating around any given hexagon. In a picture,

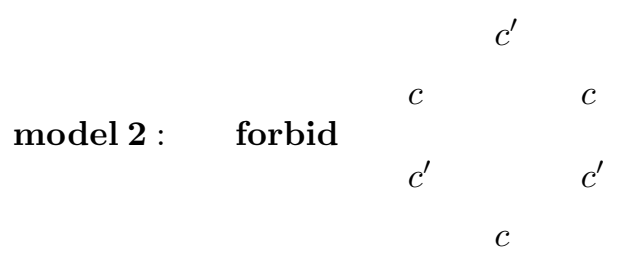

where $c \neq c^{\prime}$ can be any of $R, G$, or $B$. This forbids 6 of the 66 allowed configurations around a hexagon in the three-color model.

Imposing the constraint (2) in the fully-packed loop formulation of the three-color model forbids the shortest loops, of length 6 . The constraint is symmetric under permutations of $R, G$, and $B$, so it forbids all "short" loops, no matter which two colors are chosen to form the loops.

c. Model 3: Consider now Ising spins $s_{i}= \pm 1$ on the triangular lattice. Instead of studying a single Ising model on this lattice, we instead consider three identical Ising models, on each of the three identical triangular sublattices of the triangular lattice. This has an (Ising) ${ }^{3}$ critical point when $K=\operatorname{arcsinh}(1 / \sqrt{3}) / 2^{11}$. The domain walls for an Ising model separate unlike spins; they live on the links of the dual lattice. For each of our three Ising models on triangular lattice, its dual lattice is the honeycomb lattice made up of the sites of the other two Ising models. It is not possible for the domain walls of a given Ising model to cross or even touch, but the walls of the different decoupled models can cross and touch. Our constraint couples the three Ising models by not allowing the walls of different models to cross (although they can touch). A configuration in this model is displayed in figure 1. In terms of the spins, consider a hexagon on the triangular lattice, comprised of six sites surrounding a given site. Label the six spins around this hexagon by $s_{i}$, so that $s_{2}, s_{4}$ and $s_{6}$ are in one of the three Ising models, while $s_{1}, s_{3}$ and $s_{5}$ are in another. Denote $d_{i}=s_{i} s_{i+2}$, with the subscripts interpreted mod 6. A domain wall occurs when $d_{i}=-1$. The constraint 
that domain walls not cross in terms of these spins is then

model 3: $\quad \mathcal{C}_{j} \equiv 3-\sum_{i=1}^{6}\left(d_{i}-d_{i} d_{i+1}+d_{i} d_{i+3} / 2\right)=0$

where $j$ is the site on the triangular lattice at the center of the hexagon. The allowed domain walls inside this hexagon are

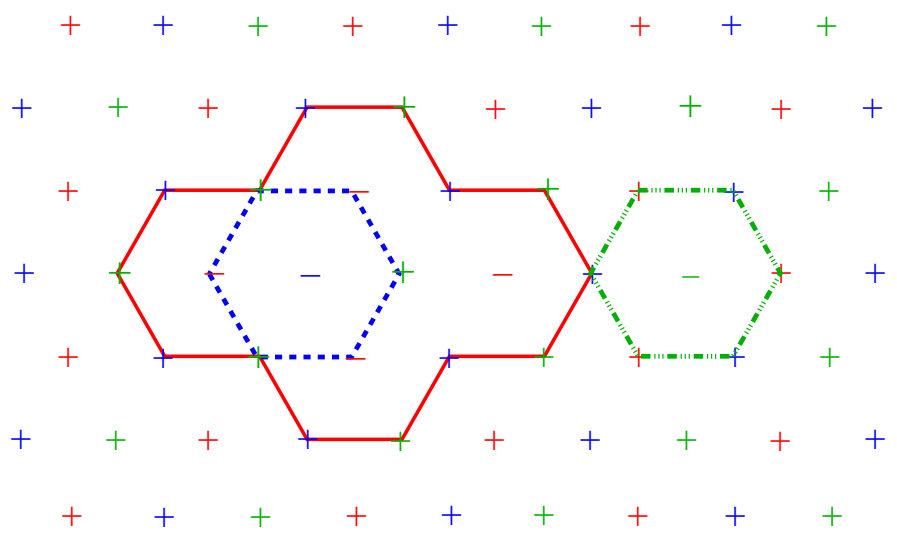

FIG. 1: Three Ising models on three triangular sublattices. The constraint (3) requires that the domain walls do not cross.

of the types illustrated in figure 3 below. Model 3 can equivalently be described in terms of closed mutually-avoiding loops on the triangular lattice, with the added restriction that loops must turn by \pm 120 degrees at every site.

These three models are equivalent to each other under local reformulations of the degrees of freedom. First let us recall the mapping of the three-color model without constraint (2) to an Ising model on the honeycomb lattice ${ }^{12}$. The Ising variables represent chiralities in the three-color model; this chirality representation occurs in the superconducting-array realization of the three-color model $1^{13,14}$. Consider given configuration in the three-color model. There are six possible configurations of the three-color model around each site of the honeycomb lattice: Put an Ising spin + on the site if the colors on the three links touching it are $R G B$ clockwise, and if the three are $R G B$ counter-clockwise. Going around each hexagon, it is easy to check that there are either 0,3 or 6 up spins. It is also easy to check for any configuration with 0,3 or 6 up spins, one can reverse the map and find a configuration in the three-color model. Ignoring boundary conditions, there are three configurations in the three-color model for each in the Ising model, so the map can be made one-to-one by specifying the color on one link. The three-color model at infinite temperature therefore maps onto the Ising model on the honeycomb lattice with $K=0$ and the requirement the sum of the $\sigma_{i}$ around hexagon obeys $M_{h}=0, \pm 6$. One can generalize the three-color model to include interactions equivalent to a non-zero $K$ if desired; this is easily done in the domain-wall formulation given below.

The equivalence of model 1 to model 2 is now obvious. Hexagons in the three-color model with alternating colors as in (2) correspond to having $M_{h}= \pm 6$ in the Ising model. These are forbidden in model 1 by (1), and in model 2 by (2).
To show the equivalence of model 1 with model 3, we reexpress the degrees of freedom in model 1 in terms of antidomain walls. Every time adjacent spins are different, we draw an antidomain wall on the link of the dual lattice separating them. These antidomain walls therefore form loops on the dual triangular lattice. Each of the configurations on each hexagon obeying the constraint (1) correspond to one of the types of antidomain-wall configurations illustrated in figure 2. There are 10 different configurations of three different types: the empty one, six (related by 60 degree rotations) with two antidomain walls, and three (related by 60 degree rotations) with four antidomain walls. Ising domain walls have a weight $e^{-2 K}$ per link. Since antidomain walls are simply the complement of the domain walls, they can be taken to have weight $e^{2 K}$ per link.

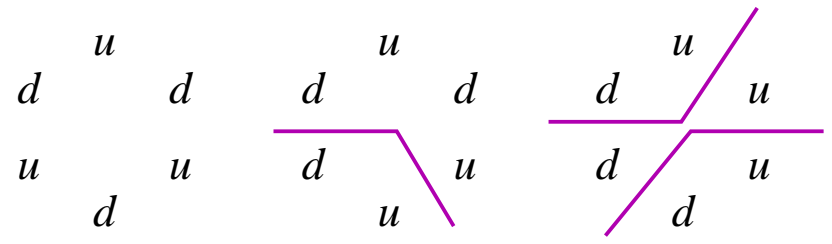

FIG. 2: The three types of antidomain-wall configurations on a hexagon in model 1 . The Ising spins here are denoted by $u$ and $d$ to distinguish them from the Ising spins in model 3, which are denoted by \pm .

These antidomain walls in model 1 correspond to domain walls in model 3. The triangular lattice for model 3 is simply the dual lattice of the honeycomb lattice for model 1 . The domain walls in a triangular-lattice Ising model make a \pm 120 degree turn at every site, just like the antidomain walls in figure 2 If the three Ising models in model 3 were decoupled, there would be 16 different domain-wall configurations going through each site of the triangular lattice, because there are four possibilities for each of the two Ising models whose domain walls go through this point. There are only 10 possibilities in figure 2. Model 1 and Model 3 are therefore equivalent

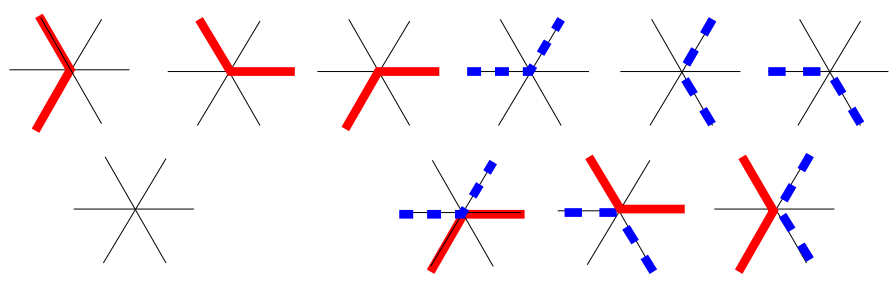

FIG. 3: The 10 possible domain-wall configurations in model 3, i.e. the 10 vertices.

if we restrict to these 10, which are redrawn in figure 3. As is obvious from the figures, the ones disallowed are those where the domain walls cross. Disallowing crossings leaves exactly the 10 , so the non-crossing constraint is the only one. In figure 1. we drew the domain walls for the three different Ising models with solid, dashed, and dot-dashed lines, to emphasize the fact that they do not cross, with each forming closed loops. In figure 3, we drew these with the dotted and dashed lines, 
but the same 10 vertices occur at any point on the triangular lattice with the appropriate types of lines. We have therefore shown that model 3 is the same as model 1, up to unimportant constants in front of the partition functions. Our model is therefore a "10-vertex model" on the triangular lattice. These vertices are a subset of those in the 32-vertex model discussed in 15 .

These proofs of course mean that model 2 is equivalent to model 3 as well, so the three-color model with and without (2) can also be written in terms of a vertex model on the triangular lattice. The usual three-color model also allows the 11th vertex pictured in figure 4. This vertex is a source/sink of domain

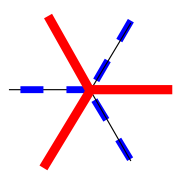

FIG. 4: The 11th vertex in the three-color model without constraint (2)

walls, and so the three-color model without constraint (2) cannot be mapped onto three Ising models. In model 1, this 11 th vertex corresponds to a hexagon with all up or all down spins, i.e. $M_{h}= \pm 6$.

In the appendix, we relate our model to two others: hard hexagons on the triangular lattice, and a generalized Ising antiferromagnet. Our model is found from these by relaxing constraints, so these models give lower bounds on the entropy of ours.

\section{CONNECTING CONFIGURATIONS BY LOCAL MOVES}

An important question in many physical applications of two-dimensional geometrically constrained models is whether the space of states is connected under local moves. It is essential if one is to study either classical or quantum dynamics, and is also useful for doing Monte Carlo simulations ${ }^{16}$. For example, to build the quantum models discussed in the introduction, without connectivity under local moves, the Hamiltonian is non-local. The three-color model is not connected: any closed loop of bonds of the honeycomb lattice that contains only two colors will give a different configuration if those two colors are permuted. Even though there exist short loops on the lattice (the shortest loop is a single hexagon), the space of states is not connected unless the dynamics is able to permute arbitrarily large loops $5,6,7$.

In this section we discuss the properties of our model under local dynamics. We show that, unlike the three-color model, the connected sectors can be enumerated simply and correspond to topological classes of sets of nonintersecting loops in the plane. Since we have shown that the constraint (2) turns the three-color model into our model, this result illuminates the reason why the three-color model is not connected by local moves.
The most-local dynamics of the Ising variables of model 1 that conserves the constraint (1) is to act on hexagons where the spins alternate up and down around the hexagon. Flipping each up spin to down and each down to up around such a hexagon preserves the constraint not only on the original hexagon, but also on each of its six neighbors as well. We display this flip in figure 5 In model 3 , this corresponds sim-

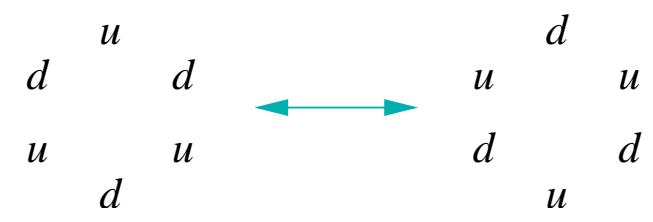

FIG. 5: The flip in terms of Ising spins in model 1

ply to flipping the Ising spin at the center of this hexagon, i.e. sending $s_{i} \rightarrow-s_{i}$.

This is the only local move necessary to connect configurations. This is easiest to see in the loop representation. Since in the model 3, the flip changes the spin at the center of this hexagon, it simply flips the model-3 loop variables on the hexagon surrounding this hexagon of model 1. An example is illustrated in figure 6 For example, if all six of the links on

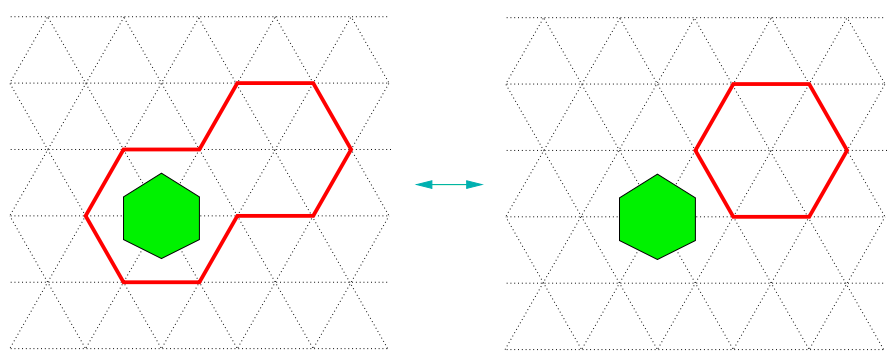

FIG. 6: An example of the effect of a flip on a loop. The alternating Ising spins in model 1 around the shaded hexagon are those flipped, as illustrated in figure 5

the surrounding hexagon are empty, the flip creates a loop of minimal length. If they are all full, this is a minimal-length loop surrounding the hexagon, and the flip removes the loop. In other cases, it shrinks or expands the loop without creating any loose ends.

It is now easy to see how the flip connects configurations. A loop of minimal length has a flippable hexagon inside it, so these can be removed by one flip. Longer loops can be shrunk and then removed by repeatedly flipping. If there are loops inside other loops, then the ones inside need to be removed first. When space is topologically a sphere, all configurations are therefore connected to the empty one. Since all processes can be reversed, this means all configurations on the sphere are connected.

When space has non-contractible cycles, however, not all loops can be removed. In order to use the formulation of model 3 , the periodic boundary conditions around a cycle must identify sites of the same triangular sublattice. When this is done, the loops are of three distinct types, as seen in figure 1 . Since flips cannot move two loops of different types through 
each other, loops which wrap around a non-contractible cycle can only be removed if they adjacent to another of the same type. The flip illustrated in figure 7 turns two adjacent noncontractible loops of the same type into two contractible ones.

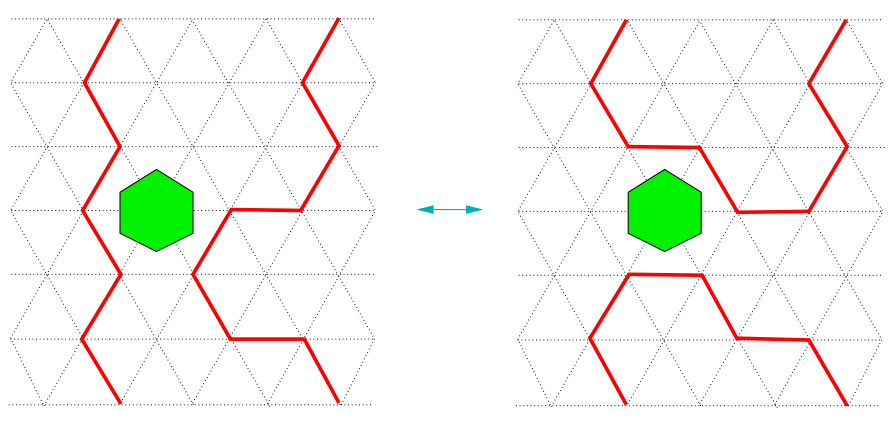

FIG. 7: A flip which converts non-contractible loops into contractible ones.

When space is a cylinder, the different sectors can be enumerated simply: a sector is given by a sequence of loop colors (those encountered reading from left to right along the cylinder, for example), with an even number of adjacent occurrences of the same colors being equivalent to the identity. Mathematically, this set is isomorphic to the free group on three elements $a, b, c$, with the relations $a^{2}=b^{2}=c^{2}=1$. Putting two cylinders next to each other defines a group action on the set of topological sectors, and this group action is nonabelian: for example, $a b a b$ is not the same as $a^{2} b^{2}=1$. Fig. 8 shows an example of how the non-intersection constraint can prevent annihilation of two loops of the same color. Each sector corresponds to a conserved charge in the transfer matrix; we will define these in section $\mathrm{V}$ When building a quantum model based on this classical model, each of these sectors will correspond to a ground state of an appropriately defined Hamiltonian. We will discuss the quantum model further in section VIII

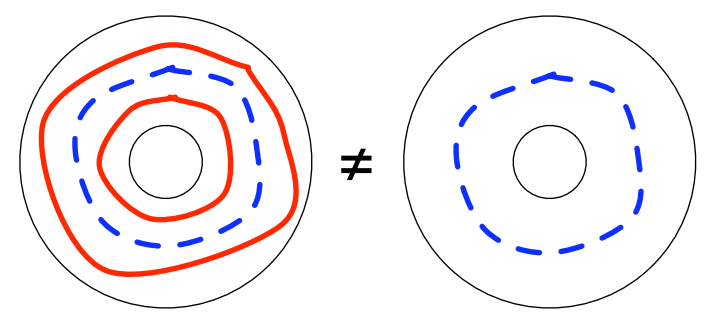

FIG. 8: Inequivalent sectors on the annulus, which is topologically equivalent to a finite cylinder. The two outer loops cannot move through the inner loop to annihilate, because of the non-intersection constraint.

Finally, when the model is defined on a torus, all noncontractible loops must go around the same cycle. This cycle can be labeled as $m \vec{c}_{1}+n \vec{c}_{2}$, where $m$ and $n$ are integers, and $\vec{c}_{1}$ and $\vec{c}_{2}$ define the torus. Most topological sectors on the torus can be labeled by $S_{c} \times S_{t}$, where $S_{c}$ is a non-trivial element of the free group defined above with the additional requirement that products must be interpreted cyclically, and $S_{t}$ is an element of $S L(2, \mathbb{Z})$, the group of modular transformations of the torus. $S L(2, \mathbb{Z})$ is generated by exchanging $m \leftrightarrow n$, and shifting $n \rightarrow n+1$. Topological sectors not of this form are the trivial sector $S_{c}=1$ (i.e. no $S_{t}$ ), and sector with a single loop (i.e. $S_{c}=a, b$ or $c$ ), where $S_{t}=\mathbb{Z}_{2} \times \mathbb{Z}_{2}$.

\section{FIELD-THEORY APPROACHES}

A basic question about our model is if it is critical. We can gain insight into this question by studying the field theories valid near two critical points which occur when by relaxing or increasing the constraints.

Three decoupled Ising models are critical when $K$ is appropriately tuned. In the continuum limit, the critical point can be described by using conformal field theory 18 . One important thing conformal field theory allows one to do is classify all the operators of the theory. The Ising model has only two relevant rotationally-invariant ones, the spin field, and the energy operator $\epsilon$. Perturbing the critical point by the latter corresponds to changing the temperature, i.e. taking $K$ away from $K_{c}$, so in the lattice model we can identify

$$
\epsilon \sim s_{i} s_{j} \sim d_{\langle i j\rangle}
$$

so that $d_{\langle i j\rangle}=-1$ corresponds to a domain wall between $i$ and $j$. A useful symmetry of the Ising model is Kramers-Wannier duality, which shows the equivalence of high- and low-temperature partition functions. In terms of the spins/fields, it takes $d \rightarrow-d$ and $\epsilon \rightarrow-\epsilon$.

To reach our model 3 , one must perturb the (Ising) ${ }^{3}$ critical point to enforce the constraint $\mathcal{C}_{j}=0$ from (3). By construction, $\mathcal{C}_{j}=0$ when the domain walls through this hexagon do not cross, and $\mathcal{C}_{j}=6$ when they cross. Thus to reduce the weight of configurations where domain walls cross, we add $\mathcal{C}_{j}$ to the energy with positive coefficient $\lambda$, i.e.

$$
E=E_{0}+\lambda \sum_{j} \mathcal{C}_{j}
$$

where $E_{0}$ is the energy of three decoupled Ising models. The constraint (3) is enforced in the $\lambda \rightarrow \infty$ limit. This perturbation is clearly relevant, since it includes the energy operators $d_{j}$ in the three individual models, and marginal terms which couple the two models. A key fact to notice is that $\mathcal{C}_{j}$ is not invariant under any of the dualities of the three Ising models.

An important result in two-dimensional statistical mechanics is the existence of a "c-theorem" that there is a function $c$ of the parameters of the theory satisfying a very important property: it cannot increase under renormalization group flows. Moreover, at a critical point its value is known from conformal field theory - it is a quantity called the central charge. Thus if one starts at a known critical point and perturbs by a relevant operator, the fact that $c$ must decrease means that either the flow must end up at a critical point with a smaller value of $c$, or at no critical point at all.

The Ising critical point has $c=1 / 2$. Since our model is a relevant perturbation of three Ising models, this implies that 
either our model is not critical, or if it is critical, it should have $c \leq 3 / 2$. The three-color model (at infinite temperature) is critical, and has $c=2^{20,21,22}$. Thus imposing constraint 2 on the three-color model should move the model away from the three-color critical point. This is in accord with the numerics discussed below.

An obvious question is if our model is critical at some value of $K$. While it is conceivable, it does not seem likely. One can cancel the relevant piece $d_{j}$ of $\mathcal{C}_{j}$ by changing the temperature of the three Ising models. This leaves the marginal terms quadratic in $d_{j}$. These marginal terms can change the dimensions of operators, so a fine-tuned model could be critical. However, since the constraint $\mathcal{C}_{j}=0$ violates dualities, this critical point is not likely to be the (Ising) ${ }^{3}$ one. This argument does not preclude a flow to a critical point with a lesser central charge.

One candidate for a flow is the hard-hexagon model. The critical point in this model has $c=4 / 5$; it is in the same universality class as the three-state Potts mode $1^{15}$. However, it occurs very far from the hard-hexagon model of interest, for several reasons. First, to get our model, one must allow configurations not present in the hard-hexagon model. Second, the latter's critical point occurs when the weight $z$ per hard hexagon is $z=z_{c} \equiv((1+\sqrt{5}) / 2)^{5}=11.09 \ldots$. To get model 3 at infinite temperature, the configurations must all be of equal weight, i.e. $z=1$. Perturbing $z$ away from $z_{c}$ is relevant. It is not clear whether allowing the additional configurations is relevant or not. It is conceivable that the two perturbations could effectively cancel, leaving one at the hardhexagon critical point, but we have no evidence for this.

There are no unitary critical points with $Z_{3}$ symmetry and $c<4 / 5$, so our model cannot be critical with these central charges. There are several with $4 / 5<c<3 / 2$, so it is conceivable that it could be critical with these central charges, but we have found no evidence for this.

\section{THE TRANSFER MATRIX}

In this section we define the transfer matrix, and show that it possesses some remarkable and unusual properties at infinite temperature. We exploit these properties in the next section to solve our model on a different lattice, and then in section VII to do numerics on very large systems.

Consider the model formulated in terms of non-crossing domain walls on the links of the triangular lattice. Take the transfer matrix to act perpendicular to one of the three axes. The transfer matrix $T$ acts on the space of states on a zig-zag line; each link is labeled by an index $i=1 \ldots 2 L$, where $L$ is the number of hexagons across the original lattice. We take the convention that links $i$ and $i+1$ meet at a vertex when $i$ is odd. The degrees of freedom are the domain walls on the links. We denote $w_{i}=1$ if there is a domain wall on link $i$, and $w_{i}=0$ if there is not. The space of states is then of dimension $2^{L}$.

The interactions are at the vertices of the lattice: the fact that there are only 10 vertices must be enforced. It is most convenient to write $T$ in the form

$$
T=U \mathcal{T} U \mathcal{T}^{-1}
$$

where $U$ moves you to the next zig-zag line, which has the property that links $i$ and $i+1$ meet at a vertex when $i$ is even. $U$ therefore imposes the weights at $L$ vertices. The operator $\mathcal{T}$ is the translation operator, which shifts all the spins by one site. The transfer matrix with periodic boundary conditions in both directions is therefore $Z=\operatorname{tr}\left(T^{M}\right)$ for a lattice of $2 L M$ hexagons. Since $T$ and $T \mathcal{T}^{2}$ have the same eigenvectors, the eigenvectors of $T$ are the same as those of $U \mathcal{T}$, and the eigenvalues are simply related.

Some interesting conservation laws follow immediately from the fact that domain walls do not cross. The total number of domain walls must be conserved $\bmod 2$, so $\sum w_{i}$ is conserved mod 2. When $N$ is a multiple of three, this conservation law is much more powerful: the transfer matrix locally conserves the number of domain walls mod 2 on each of the three sublattices. Namely, just as the sites can be divided into three sublattices, the links can as well; these result in the three types of the domain walls illustrated in figure 1 . The power of the non-crossing constraint is that adjacent domain walls of different types cannot change places or annihilate as the transfer matrix evolves the system across the lattice. The distinct sectors on the cylinder described at the end of section $\amalg$ are a consequence of this symmetry.

This symmetry is already quite powerful. By studying $U$, we find even more remarkable properties. $U$ commutes with $2 L$ local symmetry generators, so the model with transfer matrix $U$ instead of $T$ has a gauge symmetry.

There are two different types of local conservation laws. The first one is easy to see. Say two consecutive links meeting at a vertex are both occupied, i.e. $w_{2 j-1} w_{2 j}=1$. Then examine the 10 vertices in figure 3 , and take the transfer matrix to act in the vertical direction. There is only one possible vertex where both incoming links are covered, the last one drawn. This vertex has both outgoing links covered as well. Thus acting with $U$ keeps $w_{2 j-1} w_{2 j}=1$, while all other vertices have $w_{2 j-1} w_{2 j}=0$ before and after $U$ acts. Thus $Q_{j} \equiv w_{2 j-1} w_{2 j}$ is conserved by $U$ for any integer $j$.

The second local conservation law is not as obvious. It involves two adjacent vertices connected by a horizontal link. This horizontal link is of the same type as the links $2 j-1$ and $2 j+2$, so an incoming domain wall on these links can turn by 120 degrees onto the horizontal link. This conservation law arises from the facts that there are no allowed vertices which have just one or three walls touching them, and that the number of walls of a given type is conserved locally mod 2. To illustrate this, first consider the case where links $2 j-1$ and $2 j+2$ are either both occupied, or both unoccupied. Computing $U$ requires summing over the two possibilities for the horizontal link. When the horizontal link is unoccupied, the only allowed contribution to $U$ is to leave the configuration unchanged. When the horizontal link is occupied, the only allowed configuration is that both-occupied annihilates into both-unoccupied, or vice versa. Therefore $U$ here does not conserve $w_{2 j-1}$ and $w_{2 j+2}$ individually, but it does preserve the number of incoming lines mod 2. Defining 
$R_{j}=\left(2 w_{2 j-1}-1\right)\left(2 w_{2 j+2}-1\right)$, here we have $R_{j}=1$ before and after $U$ acts. When one of the two links $2 j-1$ and $2 j+2$ is occupied and the other unoccupied, $R_{j}=-1$. In this case, when the horizontal link is unoccupied, $U$ leaves the configuration unchanged, and when the horizontal link is occupied, the two configurations change place. Thus $R_{j}$ remains -1 before and after $U$ acts. Thus $R_{j}$ is a local conserved quantity. Note that $\prod_{j} R_{j}=(-1)^{W}$, where $W=\sum_{i} w_{i}$ is the total number of walls (which is indeed conserved mod 2).

The quantities $Q_{j}$ and $R_{j}$ are not conserved in the full model, because they do not commute with translation operator $\mathcal{T}$. However, in the infinite-temperature case $K=0$, they do allow the non-zero eigenvalues of the full transfer matrix $T$ to be found from much smaller matrices. For example, we show in section VII how this gives the largest eigenvalue of $T$ for $L=6$ from a 5-by-5 matrix, considerably smaller than the $2^{12} \times 2^{12}$ transfer matrix obtained without exploiting any symmetries!

The key simplification in the $K=0$ limit is that $U$ becomes a sum of projection operators. Precisely, for each set of values of the $Q_{j}$ and $R_{j}$, define a matrix $\mathcal{P}\left(\left\{Q_{j}\right\},\left\{R_{j}\right\}\right)$ acting on the $2^{2 L}$ states on the zig-zag line. The matrix elements $\mathcal{P}\left(\left\{Q_{j}\right\},\left\{R_{j}\right\}\right)_{a b}$ are defined to be 1 if both states $a$ and $b$ have the charges $\left\{Q_{j}\right\},\left\{R_{j}\right\}$, and 0 if either or both do not. Then the result is that

$$
U=\sum \mathcal{P}\left(\left\{Q_{j}\right\},\left\{R_{j}\right\}\right) \quad \text { when } K=0,
$$

where the sum is over all possible values of $Q_{j}=0,1$ and $R_{j}= \pm 1$. Note that not all values are possible: for example, if $Q_{j}=1$ and $Q_{j+1}=1$, then $R_{j}$ must be 1 as well. The decomposition (5) follows from an extension of the arguments which led to $\left[U, Q_{j}\right]=\left[U, R_{j}\right]=0$. There we saw that each initial state leads to an outgoing state with the same charges at most once. Since $K=0$, all allowed configurations have the same weight 1 , so every entry of $U$ must be 0 or 1 . Moreover, by explicitly examining all the possibilities for each set of four successive sites $2 j-1,2 j, 2 j+1,2 j+2$ and the horizontal links touching the two vertices, it is easy to see that $U$ takes any initial state with a given values of $Q_{j}, Q_{j+1}, R_{j}$ and $R_{j+1}$, to any final state with the same values. Thus $U$ indeed is block diagonal, with each block given by the operator $\mathcal{P}\left(\left\{Q_{j}\right\},\left\{R_{j}\right\}\right)$.

Let us give an explicit example with $L=2$ and periodic boundary conditions. We denote a state with domain walls at $i, j, k$ by $(i, j, k)$, and the empty state as (). Consider the sector which has have the same conserved charges as the empty state, which are $Q_{1}=Q_{2}=0$, and $R_{1}=R_{2}=1$. The other states which have these charges are $(1,4),(2,3)$. It is then easy to check that on these three states

$$
U=\mathcal{P}(\{0,0\},\{1,1\})=\left(\begin{array}{lll}
1 & 1 & 1 \\
1 & 1 & 1 \\
1 & 1 & 1
\end{array}\right)
$$

There are two states in each of the other sectors with $Q_{1}=$ $Q_{2}=0$. When $R_{1}=-R_{2}=1$, the sector is comprised of (2) and (3), when $R_{1}=-R_{2}=-1$ it consists of (1) and (4), and when $R_{1}=R_{2}=-1$, it consists of $(1,3)$ and $(2,4)$.
Within any of these sectors,

$$
U=\left(\begin{array}{ll}
1 & 1 \\
1 & 1
\end{array}\right)
$$

$U$ for each of the 7 states with $Q_{1}=1$ and/or $Q_{2}=1$ is diagonal: there is only one state in each sector.

The crucial property of $U$ at $K=0$ is that it is proportional to a projection operator. Namely, the product of two different projection operators is zero, and each $\mathcal{P} \mathcal{P}^{2}=n \mathcal{P}$, where $n$ is the number of states in this sector. Each $\mathcal{P}$ has only a single non-zero eigenvalue $n$, and the corresponding eigenstate is the equal-amplitude sum over all states in the sector. Thus most states in the Hilbert space are annihilated by $T$. The eigenstates of $T$ with non-zero eigenvalues have an important property, following from the fact that all states in the same sector end up with the same coefficient after acting with $U$. Since $U$ is the last part of of $T$, the final state after acting with $T$ must have the same property: all states with the same values of $\left\{Q_{j}\right\}$ and $\left\{R_{j}\right\}$ have the same coefficient in the end. This means that at $K=0$, all eigenstates of $T$ with non-zero eigenvalue must have the same property as well!

We can therefore work in a space of states vastly reduced in size, by keeping just one state in each sector. How to work out the explicit transfer matrix in this reduced basis is explained in section VII. We emphasize that the $Q_{j}$ and $R_{j}$ are not conserved charges for the full transfer matrix $T$, like they are for $U$. The eigenstates of $T$ do not have definite values of the $Q_{j}$ and $R_{j}$, but are a sum over states with different values. Our result here says that for eigenstates of $T$ with non-zero eigenvalues, all states in a given sector must have the same coefficient. This is not a symmetry, because the coefficients are not the same for eigenstates with zero eigenvalue.

\section{THE GAUGE-SYMMETRIC MODEL}

Since the matrix $U$ commutes with all the local symmetry generators, using it as a transfer matrix results in a model with a gauge symmetry. Because of the gauge symmetry, the resulting "model $U$ " can be reduced to a one-dimensional model and solved exactly. In this respect it is quite similar to the two-dimensional Ising gauge theory. However, the solution of model $U$ has some very striking properties of its own: the eigenvalues of the transfer matrix are given in terms of Fibonacci numbers. We derive this here.

Model $U$ is the Ising model with constraint (1) around each plaquette of the lattice pictured in figure 9 It is the square lattice, with an extra site added to all the horizontal links. It is therefore not rotationally invariant. We find explicit expressions for the eigenvalues of $U$ in the limit $K=0$, where we can exploit the fact that its transfer matrix $U$ can be written as the sum (5). This means that the eigenstates are the sum over all states in a given sector, and the corresponding eigenvalue is the number of states in that sector. This turns out to be an amusing combinatorial problem.

Let us consider the sector including the empty state, which has all $Q_{j}=0$ and all $R_{j}=1$. Having $Q_{j}=w_{2 j-1} w_{2 j}=0$ means that the links $2 j-1$ and $2 j$ are not both occupied 


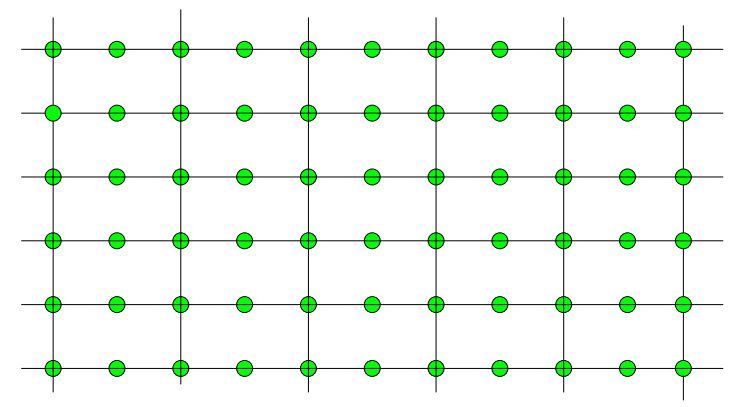

FIG. 9: The lattice for model $U$.

by walls. Having $R_{j}=1$ means that either both of the links $2 j-1$ and $2 j+2$ are occupied, are neither one is. The eigenvalue for $\mathcal{P}(\{0,0, \ldots\},\{1,1, \ldots\})$ is then the number of states $\Lambda_{0}$ satisfying these constraints. To count these, note that if both links $2 j-1$ and $2 j+2$ are occupied, then links $2 j$ and $2 j+1$ must be unoccupied, in order to preserve $R_{j}=R_{j+1}=0$. But if these latter two links are unoccupied, then links $2 j-3$ and $2 j+4$ must be unoccupied as well, to keep $Q_{j-1}=Q_{j+1}=1$. This rule gives a way of counting the configurations in this sector using one-dimensional transfer matrix $V$, which propagates the system by two sites. Start at one end. If $w_{1}=w_{4}=0$, then $w_{3}=w_{6}$ can be either 0 or 1. However, if $w_{1}=w_{4}=1$, then $w_{3}=w_{6}=0$. Iterating this procedure along the whole line gives

$$
\begin{aligned}
\Lambda_{0} & =\operatorname{tr}\left(V^{L}\right), \\
V & =\left(\begin{array}{ll}
1 & 1 \\
1 & 0
\end{array}\right)
\end{aligned}
$$

The first row and column of $V$ correspond to unoccupied links, while the second correspond to occupied ones. It is simple to show by induction that

$$
V^{a}=\left(\begin{array}{cc}
F_{a+1} & F_{a} \\
F_{a} & F_{a-1}
\end{array}\right)
$$

where $F_{a}$ is the $a$ th Fibonacci number $\left(F_{0}=0, F_{1}=1\right.$, and $F_{a}=F_{a-1}+F_{a-2}$ for the rest). Thus

$$
\Lambda_{0}=F_{L+1}+F_{L-1}
$$

which for large $L$ grows as $\tau^{L+1}$, where $\tau=(1+\sqrt{5}) / 2$ is the golden mean.

Using a transfer matrix in one dimension makes it possible to write an expression for all the eigenvalues. First consider the case with all $R_{j}=1$ except $R_{1}=-1$, and all $Q_{j}=0$. If $w_{1}=1-w_{4}=0$, then $w_{3}=1$, but if $w_{1}=1-w_{4}=1$, then $w_{3}$ can be either 0 or 1 . Thus the $1 \mathrm{~d}$ transfer matrix for $j=1$ is $V A$, where

$$
A=\left(\begin{array}{ll}
0 & 1 \\
1 & 0
\end{array}\right) .
$$

The eigenvalue $\Lambda_{1}$ for the case where one of the $R_{j}$ is flipped to -1 is therefore

$$
\Lambda_{1}=\operatorname{tr}\left(A V^{L}\right)=2 F_{L}=\Lambda_{0}-F_{L-3} .
$$

In general, when a given $R_{k}=-1$, one simply inserts $A$ at the $k$ th site. Thus if $R_{k}=R_{k+a}=-1$ with all others remaining 1 , we have eigenvalue

$$
\Lambda_{a, L-a}=\operatorname{tr}\left(A V^{a} A V^{L-a}\right) .
$$

By using various identities for Fibonacci numbers, one finds

$$
\Lambda_{a, L-a}=\Lambda_{0}-F_{L-a} F_{a}
$$

Letting some of the $Q_{j}$ be 1 can be handled in a similar fashion. As noted above, having $Q_{k}=1$ means that the walls on links $k-2$ and $k+3$ automatically follow from knowing $R_{j-1}$ and $R_{j}$. This is handled in the transfer-matrix formalism by inserting the matrix $V B$ at the site of every $Q_{k}=1$, where

$$
B=\left(\begin{array}{ll}
0 & 0 \\
0 & 1
\end{array}\right) \text {. }
$$

Thus when $Q_{k}=1$ for some $k$ while all other $Q_{j}=0$ and all $R_{j}=1$, we have eigenvalue

$$
\operatorname{tr}\left(B V^{L}\right)=F_{L-1}=\Lambda_{0}-F_{L+1}
$$

This eigenvalue is smaller than $\Lambda_{1}$ and $\Lambda_{a, L-a}$; the eigenvalue $\Lambda_{0}$ is the largest, with $\Lambda_{1}$ the next highest.

Continuing in this fashion, one obtains the general formula

$$
\begin{aligned}
\Lambda\left(\left\{Q_{j}\right\},\left\{R_{j}\right\}\right) & =\operatorname{tr}\left(V X_{L} V X_{L-1} \ldots V X_{2} V X_{1}\right) \\
X_{j} & =A^{\left(1+R_{j}\right) / 2} B^{1-Q_{j}}
\end{aligned}
$$

The conservation laws still hold when $K \neq 0$, so $U$ remains block diagonal. However, the blocks are no longer projection operators, so there is generically more than one nonzero eigenvalue per block. We suspect however that the gauge symmetry makes it possible the eigenvalues here in terms of a one-dimensional transfer matrix like 677.

\section{NUMERICAL RESULTS}

To use exact diagonalization on the transfer matrix of the full model at $K=0$, we utilize the trick described in section $\nabla$ to reduce its size. This enables us to find its largest eigenvalue for cylinders of up to $L=18$ hexagons (36 Ising sites).

Each state in this new space is labeled by the values $\left\{Q_{j}\right\}$ and $\left\{R_{j}\right\}$, which for short we call $B$. After $U \mathcal{T}$ acts, giving every element in the same block the same coefficient, we label the blocks $B^{\prime}$. To work out the transfer matrix in this new basis, first one needs to list all the states in a given block $B$. Pick one and act with $\mathcal{T}$, i.e. just shift the whole thing over by one site. Compute the new values of $\left\{Q_{j}\right\}$ and $\left\{R_{j}\right\}$ after the shift, or equivalently, compute $\widetilde{Q}_{j}=w_{2 j} w_{2 j+1}$ and $\widetilde{R}_{j}=\left(2 w_{2 j}-1\right)\left(2 w_{2 j+3}-1\right)$, which we collectively label $\widetilde{B}$. The block $B^{\prime}$ reached from acting with $U \mathcal{T}$ on this element $B$ is then labeled by $\left\{Q_{j}\right\}$ and $\left\{R_{j}\right\}$, where $Q_{j}=\widetilde{Q}_{j}$ and $R_{j}=\widetilde{R}_{j}$ for all $j$. One does this for each element in the 


\begin{tabular}{|c|c|c|c|c|c|c|}
\hline \multirow[t]{2}{*}{ Width $(\square)$} & \multicolumn{2}{|c|}{$S / \square$} & \multicolumn{2}{|c|}{$c_{\mathrm{est}}$} & \multicolumn{2}{|c|}{$f_{0, \text { est }}$} \\
\hline & Model 3 & Three-color & Model 3 & Three-color & Model 3 & Three-color \\
\hline 3 & 0.4621 & 0.4621 & & & & \\
\hline 6 & 0.3911 & 0.4028 & 1.880 & 1.569 & 0.3674 & 0.3830 \\
\hline 9 & 0.3771 & 0.3900 & 2.000 & 1.829 & 0.3659 & 0.3798 \\
\hline 12 & 0.3722 & 0.3853 & 1.990 & 1.914 & 0.3660 & 0.3793 \\
\hline 15 & 0.3700 & & 1.965 & & 0.3660 & \\
\hline 18 & 0.3688 & & 1.942 & & 0.3661 & \\
\hline$\vdots$ & $\vdots$ & $\vdots$ & & & & \\
\hline$\infty$ & 0.3661 & 0.3791 & & 1.99 & 0.3661 & 0.3791 \\
\hline Theory & & 0.379114 & & 2 & & 0.379114 \\
\hline
\end{tabular}

TABLE I: Results of numerical transfer-matrix calculations on model 3 with periodic boundary conditions, compared to three-color model. The estimated central charge $c_{\text {est }}$ and bulk free energy $f_{0, \text { est }}$ are obtained for $n$ hexagons by fitting the entropy values for $n$ and $n-3$ hexagons to equation (8). Extrapolations of entropy per site to the infinite system fit the last three points to $c_{0}+c_{2} L^{-2}+c_{4} L^{-4}$.

block $B$ : work out $\widetilde{B}$ and then $B^{\prime}$ for each, and then increase the element $\mathcal{R}_{B^{\prime} B}$ by one. Going through all the blocks gives the reduced transfer matrix $\mathcal{R}$.

Since the eigenvectors of $U \mathcal{T}$ are the same as those of $T$, and the eigenvalues are simply related, we focus on this. To give an example, for $L=2$, we have

$$
U \mathcal{T}=\left(\begin{array}{lllll}
1 & 0 & 0 & 1 & 1 \\
1 & 0 & 0 & 1 & 1 \\
1 & 0 & 0 & 1 & 1 \\
0 & 0 & 1 & 0 & 0 \\
0 & 1 & 0 & 0 & 0
\end{array}\right)
$$

which has eigenvalues $2,-1,0,0,0$. Note that it is not symmetric. There are three blocks here. The block $B=1$ has three states (), (14), (23), the block $B=2$ has just (12), and the block $B=3$ has just (34). Upon acting with $\mathcal{T}$, () goes to the block (), which means $U \mathcal{T}$ takes it to all the members of this block. Thus we increase $\mathcal{R}_{11}$ by 1 . Acting with $\mathcal{T}$ on (14) takes it to (12), so we increase $\mathcal{R}_{21}$ by 1 . Acting with $\mathcal{T}$ on (23) gives (34), so $\mathcal{R}_{31}=1$. Doing this for the other two blocks gives

$$
\mathcal{R}=\left(\begin{array}{lll}
1 & 1 & 1 \\
1 & 0 & 0 \\
1 & 0 & 0
\end{array}\right)
$$

This has eigenvalues $2,-1,0$ as we want, and is symmetric it just lost some zero eigenvalues.

The ground-state sector for $L=3$ is even easier. There are 4 states in the block 1: (), (14), (25), (36). These all go to the same block under $U \mathcal{T}$, so the reduced transfer matrix is simply a number: 4 . This is indeed the largest eigenvalue here. For higher $L$, the size of $\mathcal{R}$ still increases exponentially, but not as quickly. To give an example of how much this reduces the size of the matrix, exploiting translation invariance and parity as well means that the largest eigenvalue of $U \mathcal{T}$ for
$L=6$ is the same as that of the 5 -by-5 matrix

$$
\left(\begin{array}{lllll}
4 & 6 & 6 & 0 & 2 \\
2 & 6 & 4 & 2 & 0 \\
1 & 2 & 2 & 0 & 0 \\
0 & 1 & 0 & 0 & 0 \\
1 & 0 & 0 & 0 & 0
\end{array}\right)
$$

In this and all the examples we have examined, $\mathcal{R}$ is upper left triangular.

We have used this reduced transfer matrix for the domainwall loop representation in numerical simulations of transfer matrices with width up to 18 hexagons in model 1 (36 Ising variables). The resulting largest eigenvalues for widths that are multiples of 3 are shown in table 1 . The entropy per hexagon converges to a number right in the middle of the upper (from the three-color model) and lower (from the hardhexagon model) bounds given in (A2).

Expanding the largest eigenvalue in a power series in $1 / L$ gives additional valuable information. When the system is at a conformally invariant critical point, the subleading piece is universal and proportional to the central charge ${ }^{23}$, which must obey $c \geq 1 / 2$ in any system with positive Boltzmann weights. If the system is not critical, this piece should fall off to zero as $L \rightarrow \infty$. The precise formula for our case is

$$
f=\frac{\log \Lambda}{L}=f_{0}+\frac{\pi c}{6} \frac{\sqrt{3}}{2} \frac{1}{L^{2}}+\ldots
$$

Here $L$ is the width in hexagons, $\Lambda$ is the largest eigenvalue of $U \mathcal{T}$, and the geometrical factor $\sqrt{3} / 2$ results from the ratio between the width and length of the transfer matrix step. The resulting estimates of central charge for our model do not converge even at the largest system sizes, while for the threecolor model, extrapolation from smaller sizes gives a central charge consistent with the expected value $c=2 \underline{20,21,22}$.

The conclusion of this transfer-matrix study is that our model is most likely not described by the $c=2$ critical theory of the three-color model, even though large system sizes are 
required to see the difference. Since the central charge does not seem to be converging to anything, the numerical results are in harmony with the field-theory arguments of section IV in suggesting that our model is non-critical (i.e., has a finite correlation length). We cannot categorically rule out that it is critical with $c<2$, but have no evidence for this scenario.

\section{FURTHER DIRECTIONS: THE QUANTUM THEORY}

In this paper we discussed constrained classical lattice models. By imposing some simple constraints on Ising spins, we found a variety of intriguing properties. In particular, we showed that the space of states on the sphere is connected under local moves, and that on surfaces with non-contractible cycles, different sectors can be labeled by loop configurations. We also presented substantial (if not conclusive) evidence that the model is not critical.

In the introduction, we mentioned a quantum motivation for studying classical lattice models with constraints. The results of this paper imply that our model has the right characteristics to yield a quantum model with a topological phase, with the added intriguing possibility that the excitations have nonabelian statistics. We therefore will conclude this paper with a discussion of the quantum model in more detail.

The connection between quantum and classical models comes from a trick due to Rokhsar and Kivelson ${ }^{10}$. Let the basis elements for the Hilbert space for the quantum model consist of configurations in the two-dimensional classical lattice model. Then one can construct a quantum Hamiltonian acting on these states with a ground state consisting of a superposition of these states, with each term having an amplitude corresponding to its weight in the classical model. Correlators in the ground state of the quantum model are then related to the correlators in the classical model.

In the quantum model, one need not impose the constraints directly on the Hilbert space, but rather one can add an energy penalty for configurations which violate the constraint. The ground state then contains only configurations satisfying the constraint. Violating the constraint locally then corresponds to a quasiparticle excitation. Thus for a given classical model, one can obtain very different quantum models depending on which defects are allowed and which are not.

Let us make this explicit in terms of model 1 . Here we can take the Hilbert space to be comprised of two-state Ising variables on the sites of the honeycomb lattice. One simply imposes an energy penalty on configurations violating constraint (1), i.e. for each hexagon $h$ one includes $M_{h}^{2}$ in the Hamiltonian. The off-diagonal terms in Hamiltonian are given by flip we defined in section III as displayed in figure 5. The trick of Rokhsar and Kivelson is to add a potential which, combined with the flip, is a projector. Namely, we add a potential term which counts the number of flippable hexagons. Then the Hamiltonian is

$$
H_{1}=\sum_{h}\left(\mathcal{N}_{h}-\mathcal{F}_{h}+\left(M_{h}\right)^{2}\right)
$$

where $\mathcal{F}_{h}$ is the flip and $\mathcal{N}_{h}$ is the number of flippable plaque- ttes. It is simple to write $H_{1}$ explicitly in terms of Ising spins, but the expression is quite unwieldy. The lowest eigenvalue of $H_{1}$ is zero, since it is the sum of projectors and a positive diagonal term. The ground state on the sphere is unique, and consists of the equal-amplitude sum over all configurations satisfying constraint (1). On surfaces with contractible cycles, the local flip cannot change the sectors described above. There will be a ground state for each of these sectors, consisting of the equal-amplitude sum of all the configurations in the sector satisfying the constraint.

The reason for the interest in quantum models of this type is that they often have topological order. Topological order means that there is no local order parameter with a non-vanishing expectation value, but only non-local ones. By using the Rokhsar-Kivelson trick, it was demonstrated that a quantum eight-vertex mode ${ }^{9}$ and a quantum dimer model on the triangular lattice ${ }^{4}$ indeed have topological order. The quantum model with Hamiltonian $H_{1}$ indeed should have topological order, since the number of ground states depends on the genus of the surface, a telltale sign. One reason why models with topological order are interesting is that they can lead to excitations with fractional statistics. Non-topological solid and superfluid quantum phases near the Rokhsar-Kivelson point corresponding to our model, and possible unconventional phase transitions, are discussed using a two-component quantum height model in Ref. 17.

The excitations in the quantum model with Hamiltonian $H_{1}$ correspond to hexagons with different numbers of up and down spins. The specific $M_{h}$ we chose means the lowestenergy defects have $M_{h}= \pm 2$. These are illustrated in figure 10. In the loop language, they correspond to joining loops of
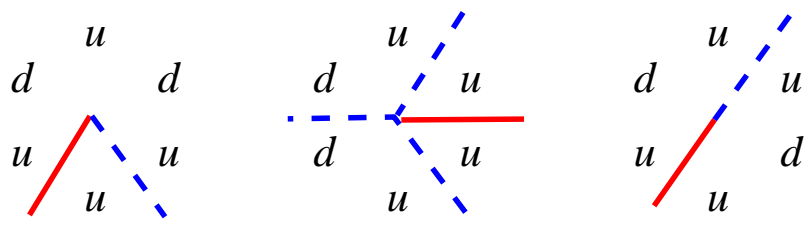

FIG. 10: Defects occurring in model 1 when $M_{h}=2$.

different types. With the Hamiltonian $H_{1}$, these defects have no dynamics, but one can of course add terms allowing them to move.

Changing the potential to favor other kinds of defects gives different theories. Allowing just $M_{h}= \pm 4$ defects gives a variation on the "odd" Ising gauge theory described in depth in ${ }^{24}$; these defects correspond to allowing loops (the domain walls of model 3) to cross. Allowing just $M_{h}= \pm 6$ defects gives a quantum version of the three-color model, i.e. the defects are the 11th vertex shown in figure 4. Note however that even though the ground states of all three models we have introduced are identical, their local defects can be quite different. The defects just described are nonlocal in the color representation of model 2 , because the three colors become rotated upon circling, i.e., each bond no longer has a uniquely defined color ${ }^{13}$. Likewise, another kind of defect we could introduce would be to treat the loops in model 3 as the degrees of freedom for the quantum model. Then we can allow defects 
to correspond to loops with ends (like the end of a flux tube in gauge theory).

The defects we have discussed have an important property: although the energy $M_{h}^{2}$ associated with them is local, they are attached to zero-energy defect lines, which can only end in another defect. For example, a $M_{h}= \pm 2$ defect has two types of domain walls attached, which must eventually end in another defect. This property makes it likely that the corresponding quasiparticles have fractional statistics, because when particles are exchanged, they must pass through these defect lines.

The $M_{h}= \pm 2$ defects illustrated in figure 10 are particularly intriguing. Since the model has an $S_{3}$ symmetry under exchange of the Ising models, these defects can be classified in representations of this non-abelian symmetry. (Note also that the larger symmetry generated by the global conserved quantities of the classical transfer matrix is non-abelian as well.) This makes it possible that a suitable choice of Hamiltonian will result in non-abelian braiding of the excitations, a topic of great current interest because of potential application to topological quantum computation ${ }^{25}$.

These arguments make it likely that one can realize a phase with topological order using our model as a starting point. To prove this, more work needs to be done. One needs to prove the quasiparticles are deconfined, i.e. that lines connecting the defects have no energy per unit length in the quantum theory. This does seem very plausible, given that $M_{h}$ is non-zero only at the location of the defect. In the three-color model, these defects have binding free energy that scales as a power-law ${ }^{13}$, which is critical between confinement and deconfinement. A related question is proving that the ground state of the Hamiltonian contains macroscopically long loops even in the continuum limit; many examples are known of lattice loop models where the average loop length (in terms of the lattice spacing) remains finite. Also, the Hamiltonian $H_{1}$ does not allow the defects and defect lines to cross through each other, making it impossible to understand the fractional statistics precisely.

We leave these very interesting open questions for future study.

We thank Ashvin Vishwanath for useful conversations. This work was supported by the NSF under grant DMR0412956 (P.F.) and DMR-0238760 (J.E.M. and C.X).

\section{APPENDIX A: RELATIONS TO OTHER MODELS}

Valuable intuition and information can be gained by relating our model to two well-studied models, the triangularlattice Ising antiferromagnet and the hard hexagon model. Our model can be found by relaxing constraints on these two. Since adding constraints reduces the entropy, the maps described in this section give lower bounds on the entropy of our model. Moreover, both have critical points different from that of the three-color model.

The Ising antiferromagnet on the triangular lattice is one of the classic examples of geometrically frustrated magnetism ${ }^{8}$. At zero temperature in the classical model, each fundamental triangle contains either two up spins and one down, or two down spins and one up. To avoid confusing these Ising spins with the earlier ones, we label them as $h_{i}= \pm 1 / 2$, so the zero-temperature constraint is that the sum of spins around every fundamental triangle is $\sum_{\triangle} h_{i}= \pm 1 / 2$. By drawing each frustrated bond as a dimer on the dual lattice, this model is identical to the close-packed hard-core dimer model on the honeycomb lattice, which is known to be critical ${ }^{26}$.

We now consider a model with the same constraint around each triangle, but where the degrees of freedom can take any half-integer value $\pm 1 / 2, \pm 3 / 2, \ldots$, not just $\pm 1 / 2$ as in the Ising antiferromagnet. We call this a "height", and prove here that this model is equivalent to ours. The Ising spin $\sigma_{i}=$ \pm 1 of our model 1 is defined on the sites of dual honeycomb lattice by

$$
\sigma_{i}=2(-1)^{i} \sum_{\triangle} h_{i}= \pm 1,
$$

where even and odd $i$ are the sites on the two equivalent sublattices of the honeycomb lattice. The constraint (1) follows automatically from this definition.

Any height configuration obeying A1 therefore defines a configuration in model 1 . To finish the proof of equivalence, we now show that each Ising spin configuration satisfying constraint (1) generates, up to two arbitrarily specified half-integers, a unique configuration of heights. Fix a configuration of Ising spins on the honeycomb lattice. Pick two adjacent sites on the dual triangular lattice, and assign them arbitrary half-integer heights $h_{1}$, and $h_{2}$. If one knows two of the heights around a triangle, and the value of the Ising spin at the center of the triangle, then A1 determines the third height uniquely. Consider the sites illustrated in figure 11 Applying (A1) to the two triangles containing both $h_{1}$ and $h_{2}$ gives the heights $h_{3}$ and $h_{4}$. Applying (A1) again to the triangles involving $\left(h_{1}, h_{3}\right)$ and $\left(h_{1}, h_{4}\right)$ gives two more heights $h_{5}$ and $h_{6}$. There is now another height $h_{7}$ which belongs to a triangle involving $\left(h_{1}, h_{5}\right)$ as well as to a triangle with $\left(h_{1}, h_{6}\right)$. Again applying (A1) to either of these triangles gives $h_{7}$; because of the constraint (1) it is determined uniquely. This therefore determines all the heights on the six triangles involving $h_{1}$. Repeating this process for the triangles around the heights $h_{2} \ldots h_{7}$ then determines the heights on another concentric ring. In this fashion all the heights follow from the Ising-spin configuration. The constraint (1) ensures that these are unique, up to the two original choices of $h_{1}$ and $h_{2}$. The indeterminacy of these two half-integers can be understood simply by noting that, if three integers $a, b, c$ are added to the height variables globally on the three sub-lattices of the triangular lattice, then as long as $a+b+c=0$, the resulting Ising spin configuration on the honeycomb lattice is unchanged.

Our model is obtained by relaxing a constraint on the zero-temperature Ising antiferromagnet, so it provides a lower bound on the entropy of our model. The honeycomb-dimer model equivalent to the former has an entropy of .323 . . per hexagon ${ }^{1,26}$. A slightly better lower bound can be obtained by relating our model to another interesting model, the hard hexagon model.

The hard hexagon model is defined by placing particles on 


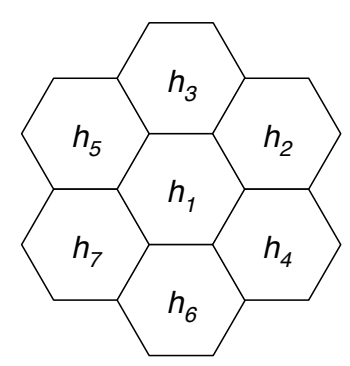

FIG. 11: The labels used in the text to establish the equivalence between model 3 and a generalization of the triangular lattice Ising antiferromagnet.

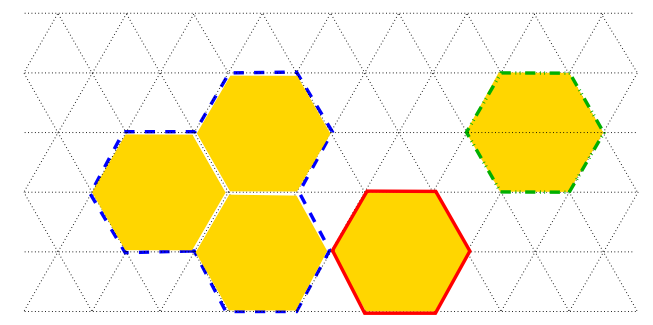

FIG. 12: A typical configuration in the hard hexagon model.

the sites of the triangular lattice, so that no two particles are adjacent or on the same site. Each particle can equivalently be viewed as a "hard" hexagon with the length of a link: the restriction that particles cannot be placed on adjacent sites means that the hexagons may not overlap ${ }^{15}$. A typical configuration is drawn in figure 12. The relation between model 3 and hard hexagons comes by drawing lines surrounding any clusters of hexagons, as shown in figure 12. Each of these loops corresponds to a domain wall in one of the three Ising models on the three triangular sublattices. By construction, these loops do not cross, although they can touch. Thus each configuration in the hard-hexagon model corresponds to one in model 3. The converse is not true: there are configurations in model 3 not in the hard hexagon model. In model 3 , one can have domain-wall loops inside of other loops, as long as they do not cross. If there is a domain-wall loop of one Ising model inside that of another, this corresponds in the hard hexagon model to placing a hexagon on top of others. This is forbidden there.

Both the hard hexagon model and the three-color model without constraint (2) are integrable. In both cases, one can compute the asymptotic behavior of the number of configurations as the number of sites gets large $e^{3.15}$. Since our model has more configurations than the hard hexagon model and less than the three-color model, this gives lower and upper bounds on the entropy $S$ in this limit:

$$
0.3332<\frac{S}{N}<0.3791
$$

where $N$ is the number of sites on the triangular lattice in model 3 (the number of hexagons in the honeycomb lattice in model 1). Our numerics discussed in section VIII give $S / N=$ $0.3661 \ldots$, consistent with these bounds.
${ }^{1}$ P.W. Kasteleyn, J. Math. Phys. 4, 287 (1963)

2 M.E. Fisher and J. Stephenson, Phys. Rev. 132, 1411 (1963)

3 R.J. Baxter, J. Math. Phys. 11, 784 (1970)

${ }^{4}$ R. Moessner and S.L. Sondhi, Phys. Rev. Lett. 86, 1881 (2001) arXiv:cond-mat/0007378

5 C.L. Henley, J. Stat. Phys. 89, 483 (1997) [arXiv:cond-mat/9607222]

6 D.A. Huse and A.P. Rutenberg, Phys. Rev. B 45, 7536 (1992)

7 C. Castelnovo, C. Chamon, C. Mudry, P. Pujol, Annals Phys. 318, 316 (2005) arXiv:cond-mat/0502068

8 P. Fazekas and P.W. Anderson, Philos. Mag. 30, 432 (1974)

9 A.Y. Kitaev, Annals of Physics 303, 2 (2003) [arXiv:quant-ph/9707021]

10 D. S. Rokhsar and S. A. Kivelson, Phys. Rev. Lett. 61, 2376 (1988)

11 B.M. McCoy and T.T. Wu, The Two-Dimensional Ising Model (Harvard, 1973).

12 P. Di Francesco and E. Guitter, Europhys. Lett. 26, 455 (1994) [arXiv:cond-mat/9402058]

13 J.E. Moore and D.-H. Lee, Phys.Rev. B 69, 104511 (2004) |arXiv:cond-mat/0309717|

14 C. Castelnovo, P, Pujol, and C. Chamon, Phys. Rev. B 69, 104529 (2004) [arXiv:cond-mat/0310710]
15 R.J. Baxter, Exactly Solved Models in Statistical Mechanics (Academic, London, 1982).

16 It is worth noting that another generalization of the hard hexagon model amenable to Monte Carlo analysis is studied in T.M. Haas, J. Stat. Phys. 54, 201 (1989)

17 C. Xu and A. Vishwanath, in preparation.

18 A. A. Belavin, A. M. Polyakov and A. B. Zamolodchikov, Nucl. Phys. B 241, 333 (1984)

19 A. B. Zamolodchikov, JETP Lett. 43, 730 (1986) [Pisma Zh. Eksp. Teor. Fiz. 43, 565 (1986)].

${ }^{20}$ N.Yu. Reshetikhin, J. Phys. A 24, 2387 (1991)

21 N. Read, reported at the Kagomé workshop (Jan. 1992).

22 J. Kondev and C.L. Henley, Nucl. Phys. B 464, 540 (1996) arXiv:cond-mat/9511102

23 H. Blöte, J. Cardy and M. Nightingale, Phys. Rev. Lett. 56, 742 (1986); I. Affleck, Phys. Rev. Lett. 56, 746 (1986).

${ }^{24}$ R. Moessner, S. L. Sondhi, and E. Fradkin Phys. Rev. B 65, 024504 (2002) |arXiv:cond-mat/0103396

25 see S. Das Sarma, M. Freedman and C. Nayak, Physics Today 59 32, (2006) and references therein.

26 F.Y. Wu, Phys. Rev. 168, 539 (1967). 